\title{
Wheeling around von Neumann-Jordan constant in Banach spaces
}

\author{
by \\ J. Alonso (Badajoz), P. Martín (Badajoz) and P. L. PAPini (Bologna)
}

\begin{abstract}
In recent times, many constants in Banach spaces have been defined and/or studied. Relations and inequalities among them (sometimes very complicated) have been indicated. But not much effort has been devoted to organize all connections, also because the literature on the subject is growing at an always bigger rate. Here we give some new connections which better the insight on some of them. In particular, we improve a known inequality between the von Neumann-Jordan and James constants.
\end{abstract}

1. Introduction. Let $X$ be a real Banach space. The so called von Neumann-Jordan constant,

$$
C_{\mathrm{NJ}}(X)=\sup \left\{\frac{\|x+y\|^{2}+\|x-y\|^{2}}{2\left(\|x\|^{2}+\|y\|^{2}\right)}: x, y \in X, \text { not both zero }\right\} \text {, }
$$

was introduced by J. A. Clarkson [3] in 1937. It was also used in [18], and then studied intensively from 1994 [13]. The paper [12] is a good reference for properties concerning this constant.

In recent times, many similar constants have been defined and/or studied in the literature, and some of them appear to be more tractable than others. Properties and relations concerning them have been proved; inequalities among them (sometimes very complicated) have been indicated. Our main aim here is to connect some results and indicate some new relations which, in our opinion, give a better understanding and clarify the role of these constants and their relationships.

The plan of this paper is as follows. In the next section we just recall a few basic definitions and related properties. In Section 3, we give some new results mainly concerning a constant, which we denote by $C_{\mathrm{NJ}}^{\prime}(X)$, and which is a slight modification of $C_{\mathrm{NJ}}(X)$. In Section 4 we obtain some

2000 Mathematics Subject Classification: 46B20, 46B99.

Key words and phrases: Banach spaces, von Neumann-Jordan constant, James constant.

Partially supported by MEC (Spain) and FEDER (UE) grant MTM2004-06226. 
facts connected with the modulus of convexity. Section 5 contains several examples. Finally, in Section 6 we discuss some relations between the value of the constants in a space and in its dual.

2. Definitions and notations. We shall denote by $S_{X}=\{x \in X$ : $\|x\|=1\}$ and $B_{X}=\{x \in X:\|x\| \leq 1\}$ the unit sphere and the unit ball of $X$. Along the paper we shall consider the following constants:

$$
\begin{aligned}
J(X) & =\sup \left\{\min \{\|x+y\|,\|x-y\|\}: x, y \in S_{X}\right\}, \\
E(X) & =\sup \left\{\|x+y\|^{2}+\|x-y\|^{2}: x, y \in S_{X}\right\}, \\
C_{\mathrm{Z}}(X) & =\sup \left\{\frac{\|x+y\|\|x-y\|}{\|x\|^{2}+\|y\|^{2}}: x, y \in X, \text { not both zero }\right\} .
\end{aligned}
$$

The constant $J(X)$, often called the James constant, have been considered in many papers (for example, [2] and [12]). The constant $E(X)$ was introduced and studied by J. Gao $[8,9,10]$. The last constant was introduced by G. Zbăganu [23], who conjectured that $C_{\mathrm{Z}}(X)$ and $C_{\mathrm{NJ}}(X)$ always coincide, which was disproved in [1] (see also [21]).

3. The von Neumann-Jordan constant in the unit sphere. The constant $E(X)$ appears in several recent papers; for example, $E(X) / 2$ was considered in [20] and [22], where it was denoted by $\gamma(1)$.

Now we shall consider the constant

$$
C_{\mathrm{NJ}}^{\prime}(X)=\sup \left\{\frac{\|x+y\|^{2}+\|x-y\|^{2}}{4}: x, y \in S_{X}\right\},
$$

i.e., $C_{\mathrm{NJ}}^{\prime}(X)=E(X) / 4$. Observe that since the function $t \in \mathbb{R} \mapsto\|x+t y\|^{2}+$ $\|x-t y\|^{2}$ is even and convex, it is increasing in $[0,1]$. Consequently, in (1) we can change $S_{X}$ to $B_{X}$. However, Example 1 shows that to compute $C_{\mathrm{NJ}}^{\prime}(X)$ we cannot consider only vectors $x, y \in S_{X}$ such that $\|x+y\|=\|x-y\|$, as is the case for $J(X)$ [17]. It is clear that for any Banach space $X$,

$$
1 \leq C_{\mathrm{NJ}}^{\prime}(X) \leq C_{\mathrm{NJ}}(X) \leq 2 .
$$

The origin of the von Neumann-Jordan constant is in the well known characterization of inner product spaces, "the parallelogram law", from which it follows that

$$
X \text { is a Hilbert space } \Leftrightarrow C_{\mathrm{NJ}}(X)=1 .
$$

From the weaker characterization given by M. M. Day [5], "the rhombus law", one finds that also

$$
X \text { is a Hilbert space } \Leftrightarrow C_{\mathrm{NJ}}^{\prime}(X)=1 .
$$

Recall that a space $X$ is called uniformly non-square, (UNS) for short, if there exists some $\varepsilon>0$ such that $\min \{\|x-y\|,\|x+y\|\} \leq 2-\varepsilon$ for all 
$x, y \in S_{X}$; equivalently, if $J(X)<2$. It is known, and easy to see, that

$$
X \text { is }(\mathrm{UNS}) \Leftrightarrow C_{\mathrm{NJ}}(X)<2 \Leftrightarrow C_{\mathrm{NJ}}^{\prime}(X)<2 .
$$

Examples 1 and 2 below (see table) show that, in general, $C_{\mathrm{NJ}}^{\prime}(X)<$ $C_{\mathrm{NJ}}(X)$. So we can ask how big the difference between the two constants can be. The next results give an answer to this question.

Theorem 1. For any Banach space $X$,

$$
C_{\mathrm{NJ}}(X) \leq 2\left(1+C_{\mathrm{NJ}}^{\prime}(X)-\sqrt{2 C_{\mathrm{NJ}}^{\prime}(X)}\right) \leq 2 .
$$

Proof. In the definition of $C_{\mathrm{NJ}}(X)$ we can assume without loss of generality that $0 \leq\|y\| \leq\|x\|=1$. Let us first consider the case where $0<\|y\| \leq\|x\|=1$. Since

$$
\left\|x \pm \frac{y}{\|y\|}\right\|=\left\|\frac{x}{\|y\|} \pm \frac{y}{\|y\|}+x-\frac{x}{\|y\|}\right\| \geq\left|\frac{\|x \pm y\|+\|y\|-1}{\|y\|}\right|,
$$

we obtain

$$
\begin{aligned}
C_{\mathrm{NJ}}^{\prime}(X) & \geq \frac{\left\|x+\frac{y}{\|y\|}\right\|^{2}+\left\|x-\frac{y}{\|y\|}\right\|^{2}}{4} \\
& \geq \frac{(\|x+y\|+\|y\|-1)^{2}+(\|x-y\|+\|y\|-1)^{2}}{4\|y\|^{2}},
\end{aligned}
$$

and from the inequality

$$
\|x+y\|+\|x-y\| \leq \sqrt{2} \sqrt{\|x+y\|^{2}+\|x-y\|^{2}}
$$

we obtain

$$
\begin{aligned}
4\|y\|^{2} C_{\mathrm{NJ}}^{\prime}(X) \geq & (\|x+y\|+\|y\|-1)^{2}+(\|x-y\|+\|y\|-1)^{2} \\
= & \|x+y\|^{2}+\|x-y\|^{2}+2(\|y\|-1)^{2} \\
& +2(\|y\|-1)(\|x+y\|+\|x-y\|) \\
\geq & \|x+y\|^{2}+\|x-y\|^{2}+2(\|y\|-1)^{2} \\
& +2(\|y\|-1) \sqrt{2} \sqrt{\|x+y\|^{2}+\|x-y\|^{2}} \\
= & \left(\sqrt{\|x+y\|^{2}+\|x-y\|^{2}}+\sqrt{2}(\|y\|-1)\right)^{2} .
\end{aligned}
$$

Therefore,

$$
2\|y\| \sqrt{C_{\mathrm{NJ}}^{\prime}(X)} \geq \sqrt{\|x+y\|^{2}+\|x-y\|^{2}}+\sqrt{2}(\|y\|-1),
$$

and so

$$
\frac{\|y\| \sqrt{2 C_{\mathrm{NJ}}^{\prime}(X)}+1-\|y\|}{\sqrt{1+\|y\|^{2}}} \geq \sqrt{\frac{\|x+y\|^{2}+\|x-y\|^{2}}{2\left(1+\|y\|^{2}\right)}} .
$$


Since the function

$$
f(t)=\frac{t \sqrt{2 C_{\mathrm{NJ}}^{\prime}(X)}+1-t}{\sqrt{1+t^{2}}}, \quad 0 \leq t \leq 1,
$$

attains its maximum at $\bar{t}=\sqrt{2 C_{\mathrm{NJ}}^{\prime}(X)}-1$, from (6) we get

$$
\begin{aligned}
\sqrt{2\left(1+C_{\mathrm{NJ}}^{\prime}(X)-\sqrt{2 C_{\mathrm{NJ}}^{\prime}(X)}\right)} & =f(\bar{t}) \\
& \geq f(\|y\|) \geq \sqrt{\frac{\|x+y\|^{2}+\|x-y\|^{2}}{2\left(1+\|y\|^{2}\right)}} .
\end{aligned}
$$

The last inequality is also true for $\|y\|=0$. So, since $x$ and $y$ are arbitrary, we obtain the left inequality in (5). The right one is equivalent to $C_{\mathrm{NJ}}^{\prime}(X) \leq 2$.

Corollary 1. For any Banach space $X$,

$$
C_{\mathrm{NJ}}(X) / 2+\sqrt{C_{\mathrm{NJ}}(X)-1} \leq C_{\mathrm{NJ}}^{\prime}(X) .
$$

Proof. Let us consider the polynomial $g(t)=2-C_{\mathrm{NJ}}(X)-2 \sqrt{2} t+2 t^{2}$, whose roots are $\left(1 \pm \sqrt{C_{\mathrm{NJ}}(X)-1}\right) / \sqrt{2}$. From Theorem 1 we know that $g\left(\sqrt{C_{\mathrm{NJ}}^{\prime}(X)}\right) \geq 0$. Therefore, either

$$
\sqrt{C_{\mathrm{NJ}}^{\prime}(X)} \leq\left(1-\sqrt{C_{\mathrm{NJ}}(X)-1}\right) / \sqrt{2}
$$

or

$$
\sqrt{C_{\mathrm{NJ}}^{\prime}(X)} \geq\left(1+\sqrt{C_{\mathrm{NJ}}(X)-1}\right) / \sqrt{2}
$$

The first case is impossible because $\left(1-\sqrt{C_{\mathrm{NJ}}(X)-1}\right) / \sqrt{2}<1$. The second case gives (7).

Corollary 2. For any Banach space $X$,

$$
C_{\mathrm{NJ}}(X)-C_{\mathrm{NJ}}^{\prime}(X) \leq 3-2 \sqrt{2} .
$$

Proof. Let $h(t)=2-2 \sqrt{2 t}+t$. From Theorem 1 we know that

$$
\begin{aligned}
C_{\mathrm{NJ}}(X)-C_{\mathrm{NJ}}^{\prime}(X) & \leq 2-2 \sqrt{2 C_{\mathrm{NJ}}^{\prime}(X)}+C_{\mathrm{NJ}}^{\prime}(X) \\
& =h\left(C_{\mathrm{NJ}}^{\prime}(X)\right) .
\end{aligned}
$$

Since $h(t)$ is decreasing in $[1,2]$, we have $h\left(C_{\mathrm{NJ}}^{\prime}(X)\right) \leq h(1)=3-2 \sqrt{2}$, which proves (8).

We note that the estimates given by Theorem 1 and Corollary 1 are sharp. The inequalities in (5) and (7) are equalities if $X$ is not (UNS). The left inequality in (5) and inequality in (7) are also equalities if $X$ is the space of Example 2 below. However, note that the estimate (7) is weak for $C_{\mathrm{NJ}}(X)$ small; in fact, if $C_{\mathrm{NJ}}(X) \leq 4-2 \sqrt{2}$ then $C_{\mathrm{NJ}}(X) / 2+\sqrt{C_{\mathrm{NJ}}(X)-1} \leq 1$ and the inequality ( 7 ) is obvious. We do not know if the estimate in Corollary 2 is sharp. 
It is immediate to see that $C_{\mathrm{NJ}}(X)$ can also be defined as

$$
C_{\mathrm{NJ}}(X)=\sup \left\{\frac{\|x+t y\|^{2}+\|x-t y\|^{2}}{2\left(1+t^{2}\right)}: x, y \in S_{X}, t \in[0,1]\right\},
$$

or, following S. Saejung [19], as $C_{\mathrm{NJ}}(X)=\sup \left\{C_{\mathrm{NJ}}(t, X): t \in[0,1]\right\}$, where

$$
C_{\mathrm{NJ}}(t, X)=\sup \left\{\frac{\|x+t y\|^{2}+\|x-t y\|^{2}}{2\left(1+t^{2}\right)}: x, y \in S_{X}\right\} \text {. }
$$

It was proved by C. Yang and F. Wang [22] that the function

$$
t \in[0,1] \mapsto \gamma_{X}(t):=\sup \left\{\frac{\|x+t y\|^{2}+\|x-t y\|^{2}}{2}: x, y \in S_{X}\right\}
$$

is continuous in $[0,1]$. Hence, the function

$$
t \in[0,1] \mapsto C_{\mathrm{NJ}}(t, X)=\frac{\gamma_{X}(t)}{1+t^{2}}
$$

is also continuous in $[0,1]$. Therefore there exists $t_{X} \in[0,1]$ such that

$$
C_{\mathrm{NJ}}(X)=C_{\mathrm{NJ}}\left(t_{X}, X\right) .
$$

Hence $C_{\mathrm{NJ}}(X)$ is always attained at some $t$ (even if $\operatorname{dim} X=+\infty$ ). But there are spaces where $C_{\mathrm{NJ}}(X)$ is attained at several $t$ : in Hilbert spaces it is attained for any $t \in[0,1]$ and in the space of Example 4 it is attained for $t=1$ and $t=\sqrt{2}-1$. The next proposition gives some bounds for $t_{X}$.

Proposition 1. For any Banach space $X$,

$$
t_{X} \geq \max \left\{\frac{1-\sqrt{2 C_{\mathrm{NJ}}(X)-C_{\mathrm{NJ}}^{2}(X)}}{C_{\mathrm{NJ}}(X)-1}, \frac{C_{\mathrm{NJ}}(X)-1}{C_{\mathrm{NJ}}(X)}\right\},
$$

where the first term should be considered equal to 0 if $C_{\mathrm{NJ}}(X)=1$.

Proof. First observe that if $C_{\mathrm{NJ}}(X)=1$, then $X$ is a Hilbert space and (10) says that $t_{X} \geq 0$, as we already know. So we can assume that $C_{\mathrm{NJ}}(X)>1$. For any $x, y \in S_{X}$,

$$
\frac{\left\|x+t_{X} y\right\|^{2}+\left\|x-t_{X} y\right\|^{2}}{2\left(1+t_{X}^{2}\right)} \leq \frac{\left(1+t_{X}\right)^{2}}{1+t_{X}^{2}} .
$$

Then

$$
C_{\mathrm{NJ}}(X)=C_{\mathrm{NJ}}\left(t_{X}, X\right) \leq \frac{\left(1+t_{X}\right)^{2}}{1+t_{X}^{2}}
$$

from which it follows that $\left(C_{\mathrm{NJ}}(X)-1\right) t_{X}^{2}-2 t_{X}+C_{\mathrm{NJ}}(X)-1 \leq 0$. By considering the interval where the polynomial $\left(C_{\mathrm{NJ}}(X)-1\right) t^{2}-2 t+C_{\mathrm{NJ}}(X)$ -1 is negative, one sees that $t_{X} \geq\left(1-\sqrt{2 C_{\mathrm{NJ}}(X)-C_{\mathrm{NJ}}^{2}(X)}\right) /\left(C_{\mathrm{NJ}}(X)-1\right)$. 
Let now $x, y \in S_{X}$. Since the function $f(t)=\|x+t y\|^{2}+\|x-t y\|^{2}$ is convex we see that

$$
\begin{aligned}
f\left(t_{X}\right) & =f\left(t_{X} \cdot 1+\left(1-t_{X}\right) \cdot 0\right) \leq t_{X} f(1)+\left(1-t_{X}\right) f(0) \\
& =t_{X}\left(\|x+y\|^{2}+\|x-y\|^{2}\right)+2\left(1-t_{X}\right) .
\end{aligned}
$$

Therefore,

$$
\begin{aligned}
\frac{\left\|x+t_{X} y\right\|^{2}+\left\|x-t_{X} y\right\|^{2}}{2\left(1+t_{X}^{2}\right)} & \leq \frac{t_{X}\left(\|x+y\|^{2}+\|x-y\|^{2}\right)+2\left(1-t_{X}\right)}{2\left(1+t_{X}^{2}\right)} \\
& =\frac{2 t_{X}}{1+t_{X}^{2}}\left(\frac{\|x+y\|^{2}+\|x-y\|^{2}}{4}\right)+\frac{1-t_{X}}{1+t_{X}^{2}} \\
& \leq \frac{2 t_{X}}{1+t_{X}^{2}} C_{\mathrm{NJ}}(X)+\frac{1-t_{X}}{1+t_{X}^{2}},
\end{aligned}
$$

and since $x$ and $y$ are arbitrary,

$$
C_{\mathrm{NJ}}(X) \leq \frac{2 t_{X}}{1+t_{X}^{2}} C_{\mathrm{NJ}}(X)+\frac{1-t_{X}}{1+t_{X}^{2}}
$$

which implies that $C_{\mathrm{NJ}}(X) t_{X}^{2}-\left(2 C_{\mathrm{NJ}}(X)-1\right) t_{X}+C_{\mathrm{NJ}}(X)-1 \leq 0$. Similarly to the above case, this implies that $t_{X} \geq\left(C_{\mathrm{NJ}}(X)-1\right) / C_{\mathrm{NJ}}(X)$.

REMARK 1. The two functions of $C_{\mathrm{NJ}}$ considered in Proposition 1 are increasing; they meet for $C_{\mathrm{NJ}}=1$ and $C_{\mathrm{NJ}}=(2+\sqrt{2}) / 2$; and $(1-$ $\left.\sqrt{2 C_{\mathrm{NJ}}-C_{\mathrm{NJ}}^{2}}\right) /\left(C_{\mathrm{NJ}}-1\right)>\left(C_{\mathrm{NJ}}-1\right) / C_{\mathrm{NJ}}$ for $C_{\mathrm{NJ}}>(2+\sqrt{2}) / 2$. Proposition 1 also shows that if $X$ is not (UNS) then $t_{X}=1$. If we also consider $C_{\mathrm{NJ}}^{\prime}$, then the proof of Proposition 1 gives us more information about $t_{X}$. In fact, we have shown that

$$
C_{\mathrm{NJ}} \leq \frac{2 t_{X}}{1+t_{X}^{2}} C_{\mathrm{NJ}}^{\prime}+\frac{1-t_{X}}{1+t_{X}^{2}}
$$

which implies that $C_{\mathrm{NJ}} t_{X}^{2}-\left(2 C_{\mathrm{NJ}}^{\prime}-1\right) t_{X}+C_{\mathrm{NJ}}-1 \leq 0$, and so

$$
0 \leq \frac{2 C_{\mathrm{NJ}}^{\prime}-1-p}{2 C_{\mathrm{NJ}}} \leq t_{X} \leq \frac{2 C_{\mathrm{NJ}}^{\prime}-1+p}{2 C_{\mathrm{NJ}}} \leq 1,
$$

where $p=\sqrt{4\left(C_{\mathrm{NJ}}^{\prime 2}-C_{\mathrm{NJ}}^{2}+C_{\mathrm{NJ}}-C_{\mathrm{NJ}}^{\prime}\right)+1}$. Since $C_{\mathrm{NJ}}^{\prime} \leq C_{\mathrm{NJ}}$ we have $\left(2 C_{\mathrm{NJ}}^{\prime}-1-p\right) /\left(2 C_{\mathrm{NJ}}\right) \geq\left(C_{\mathrm{NJ}}-1\right) / C_{\mathrm{NJ}}$, which improves one of the bounds in $(10)$.

4. The role of the modulus of convexity. The modulus of convexity of a normed space $X$ is defined for $\varepsilon \in[0,2]$ as

$$
\delta_{X}(\varepsilon)=\inf \left\{1-\|x+y\| / 2: x, y \in S_{X},\|x-y\| \geq \varepsilon\right\},
$$

where " $S_{X}$ " and " $\geq$ " can be (equivalently) replaced by " $B_{X}$ " and "=" [4]. Next we recall some results depending on this modulus. 
Obviously, the modulus of convexity is a nondecreasing function in $[0,2]$. Moreover, the function $\varepsilon \in(0,2] \mapsto \delta_{X}(\varepsilon) / \varepsilon$ is also nondecreasing [6].

A normed space $X$ is (UNS) (equivalently, $J(X)<2$ ) if and only if $\delta_{X}(\varepsilon)>0$ for some $0<\varepsilon<2$; and it is strictly convex if and only if $\delta_{X}(2)=1$.

Proposition 2 ([2, Remark 2.4]). If $X$ is (UNS) then

$$
J(X)=2(1-\delta(J(X))) .
$$

Note that the equality (12) can fail to hold if $X$ is not (UNS). Consider, for example, the space $C(I)$ with the norm $\|f\|=\|f\|_{2}+\|f\|_{\infty}$, which is strictly convex but not (UNS).

Proposition 3 ([11, Theorem 3.1]). For any normed space $X$ and $0<$ $\varepsilon \leq \varepsilon^{\prime} \leq 2$,

$$
\frac{\delta_{X}\left(\varepsilon^{\prime}\right)-\delta_{X}(\varepsilon)}{\varepsilon^{\prime}-\varepsilon} \leq \frac{1-\delta_{X}(\varepsilon)}{2-\varepsilon} .
$$

Proposition 4. For any normed space $X$,

$$
C_{\mathrm{NJ}}^{\prime}(X)=\sup \left\{\varepsilon^{2} / 4+\left(1-\delta_{X}(\varepsilon)\right)^{2}: 0 \leq \varepsilon \leq 2\right\} .
$$

Proof. Let $K=\sup \left\{\varepsilon^{2} / 4+\left(1-\delta_{X}(\varepsilon)\right)^{2}: 0 \leq \varepsilon \leq 2\right\}$. For $x, y \in S_{X}$ we have $\delta_{X}(\|x-y\|) \leq 1-\|x+y\| / 2$. Then

$$
\frac{\|x+y\|^{2}+\|x-y\|^{2}}{4} \leq \frac{\|x-y\|^{2}}{4}+\left(1-\delta_{X}(\|x-y\|)\right)^{2} \leq K,
$$

from which it follows that $C_{\mathrm{NJ}}^{\prime}(X) \leq K$. On the other hand, let $0 \leq \varepsilon \leq 2$. Then, for any $\mu>0$ there exist $x, y \in S$ such that $\|x-y\|=\varepsilon$ and $1-$ $\|x+y\| / 2 \leq \delta_{X}(\varepsilon)+\mu$. Hence

$$
C_{\mathrm{NJ}}^{\prime}(X) \geq \frac{\|x+y\|^{2}+\|x-y\|^{2}}{4} \geq \frac{\varepsilon^{2}}{4}+\left(1-\delta_{X}(\varepsilon)-\mu\right)^{2} .
$$

Since $\mu$ is arbitrary, we obtain $C_{\mathrm{NJ}}^{\prime}(X) \geq K$, which completes the proof.

With the help of the above results we are ready to prove one of our main results.

Theorem 2. For any normed space $X$,

$$
C_{\mathrm{NJ}}^{\prime}(X) \leq J(X)
$$

Proof. From (14) it is enough to see that for every $0 \leq \varepsilon \leq 2$,

$$
\varepsilon^{2} / 4+\left(1-\delta_{X}(\varepsilon)\right)^{2} \leq J(X) .
$$

Recall that $\sqrt{2} \leq J(X) \leq 2$. We can assume that $J(X)<2$ because otherwise $J(X)=2=C_{\mathrm{NJ}}^{\prime}(X)$ and (15) trivially holds. Let

$$
\alpha=(2 \sqrt{J(X)-1}) / J(X) .
$$


Then

$$
0<\alpha J(X)<J(X)<\sqrt{4 J(X)-J(X)^{2}}<2 .
$$

To prove (16) we shall consider several cases according to the location of $\varepsilon$ in the subintervals of $[0,2]$ defined by the above inequalities. For simplicity we shall denote $C_{\mathrm{NJ}}^{\prime}(X), J(X)$ and $\delta_{X}$ by $C^{\prime}, J$ and $\delta$, respectively.

CASE 1. Assume that $0 \leq \varepsilon \leq \alpha J$. Then

$$
\frac{\varepsilon^{2}}{4}+(1-\delta(\varepsilon))^{2} \leq \frac{\alpha^{2} J^{2}}{4}+(1-\delta(\varepsilon))^{2} \leq \frac{\alpha^{2} J^{2}}{4}+1=J .
$$

CASe 2. Assume that $\alpha J \leq \varepsilon \leq J$. From (13) we know that

$$
\frac{\delta(J)-\delta(\alpha J)}{J-\alpha J} \leq \frac{1-\delta(\alpha J)}{2-\alpha J},
$$

which, jointly with (12), implies that

$$
\delta(\alpha J) \geq \frac{\alpha J(1-\delta(J))+2 \delta(J)-J}{2-J}=\frac{J \sqrt{J-1}+2-2 J}{2-J} .
$$

Since $\delta_{X}$ is a nondecreasing function, the above implies that

$$
\frac{\varepsilon^{2}}{4}+(1-\delta(\varepsilon))^{2} \leq \frac{J^{2}}{4}+(1-\delta(\alpha J))^{2} \leq \frac{J^{2}}{4}+\left(\frac{J-J \sqrt{J-1}}{2-J}\right)^{2} .
$$

Now we shall see that the last term above is less than or equal to $J$. Since $2 J-2 \leq \sqrt{(2 J-2)^{2}+(J-1)(J-2)^{2}}=J \sqrt{J-1}$, we have

$$
0 \leq \frac{J-J \sqrt{J-1}}{2-J} \leq 1
$$

and therefore

$$
\begin{aligned}
\frac{J^{2}}{4}+\left(\frac{J-J \sqrt{J-1}}{2-J}\right)^{2} & \leq \frac{J^{2}}{4}+\frac{J-J \sqrt{J-1}}{2-J} \\
& =J\left(\frac{J}{4}+\frac{1-\sqrt{J-1}}{2-J}\right) .
\end{aligned}
$$

It only remains to see that $\frac{J}{4}+\frac{1-\sqrt{J-1}}{2-J} \leq 1$, which is equivalent to $J^{2}-6 J+$ $4 \sqrt{J-1}+4 \geq 0$. But

$$
\begin{aligned}
J^{2}-6 J+ & 4 \sqrt{J-1}+4 \\
= & (1-\sqrt{2}+\sqrt{J-1})(1+\sqrt{2}+\sqrt{J-1})(1-\sqrt{J-1})^{2} \geq 0,
\end{aligned}
$$

because $\sqrt{J-1} \geq \sqrt{\sqrt{2}-1} \geq \sqrt{2}-1$.

CAsE 3. Assume that $J \leq \varepsilon \leq \sqrt{4 J-J^{2}}$. Then

$$
\frac{\varepsilon^{2}}{4}+(1-\delta(\varepsilon))^{2} \leq \frac{\varepsilon^{2}}{4}+(1-\delta(J))^{2}=\frac{\varepsilon^{2}}{4}+\frac{J^{2}}{4} \leq \frac{4 J-J^{2}}{4}+\frac{J^{2}}{4}=J .
$$


CASe 4. Assume that $\sqrt{4 J-J^{2}} \leq \varepsilon \leq 2$. Then, since

$$
\frac{2-J}{2 J}=\frac{\delta(J)}{J} \leq \frac{\delta\left(\sqrt{4 J-J^{2}}\right)}{\sqrt{4 J-J^{2}}},
$$

we obtain

$$
\begin{aligned}
\frac{\varepsilon^{2}}{4}+(1-\delta(\varepsilon))^{2} & \leq 1+(1-\delta(\varepsilon))^{2} \leq 1+\left(1-\delta\left(\sqrt{4 J-J^{2}}\right)\right)^{2} \\
& \leq 1+\left(1-\frac{(2-J) \sqrt{4 J-J^{2}}}{2 J}\right)^{2}
\end{aligned}
$$

and the last term above is less than or equal to $J$ if and only if

$$
(2-J)\left(J^{2}-2 J+8-4 \sqrt{4 J-J^{2}}\right) \leq 0 .
$$

Finally, $J^{2}-2 J+8-4 \sqrt{4 J-J^{2}}=\left(J^{2}-3 J+2\right)+\left(J+6-4 \sqrt{4 J-J^{2}}\right) \leq 0$ because $t^{2}-3 t+2 \leq 0$ for $t \in[1,2]$ and $t+6-4 \sqrt{4 t-t^{2}} \leq 0$ for $t \in\left[\frac{18}{17}, 2\right]$, and $J$ belongs to both intervals.

Question 1. In all examples we know, the inequality $C_{\mathrm{NJ}}(X) \leq J(X)$ holds. Does it hold for any space? Does equality hold only when both constants are equal to 2 ?

It is not difficult to see that the inequality

$$
C_{\mathrm{NJ}}^{\prime}(X) \leq 1+J(X)^{2} / 4
$$

holds for any normed space $X$, and it has been an open problem if the analogous inequality is valid for $C_{\mathrm{NJ}}(X)$ instead of $C_{\mathrm{NJ}}^{\prime}(X)$. Some weaker inequalities have been proved in [16], while a solution of the problem has been claimed in [19], but with a doubtful proof. The next theorem answers the problem in the affirmative, giving an even sharper inequality. It is based on the inequality (5), which in fact improves (17).

TheOREM 3. For any normed space $X$,

$$
C_{\mathrm{NJ}}(X) \leq 2(1+J(X)-\sqrt{2 J(X)}) .
$$

Proof. From (5) we know that $C_{\mathrm{NJ}}(X) \leq 2\left(1+C_{\mathrm{NJ}}^{\prime}(X)-\sqrt{2 C_{\mathrm{NJ}}^{\prime}(X)}\right)$. The function $t \in(1,2) \mapsto 2(1+t-\sqrt{2 t})$ is increasing, so (18) follows from (15).

Remark 2. Since $J=J(X) \geq \sqrt{2}$, we have

$$
\begin{aligned}
2(1+J-\sqrt{2 J}) & =1+\frac{J^{2}}{4}-\frac{1}{4}(\sqrt{J}-2+\sqrt{2})(\sqrt{J}+2+\sqrt{2})(\sqrt{2}-\sqrt{J})^{2} \\
& \leq 1+\frac{J^{2}}{4}
\end{aligned}
$$

as anticipated. Also note that the bound in (18) is sharp in non-(UNS) spaces. 
With regard to the lower bounds of $C_{\mathrm{NJ}}(X)$ it is well known (see [12]) that $J(X)^{2} / 2 \leq C_{\mathrm{NJ}}(X)$. The next proposition shows that the same bound is valid for $C_{\mathrm{NJ}}^{\prime}(X)$, and is also sharp in non-(UNS) spaces.

Proposition 5. For any normed space $X$,

$$
J(X)^{2} / 2 \leq C_{\mathrm{NJ}}^{\prime}(X) .
$$

Proof. From (14) and (12) it follows that

$$
C_{\mathrm{NJ}}^{\prime}(X) \geq J(X)^{2} / 4+\left(1-\delta_{X}(J(X))\right)^{2}=J(X)^{2} / 2 .
$$

With regard to $C_{\mathrm{Z}}(X)$, recall that for any normed space $X$ one has $1 \leq C_{\mathrm{Z}}(X) \leq C_{\mathrm{NJ}}(X) \leq 2$, but it seems that there is no apparent relation between this constant and $C_{\mathrm{NJ}}^{\prime}(X)$. In fact, for inner product spaces and for non-(UNS) spaces one has $C_{\mathrm{Z}}(X)=C_{\mathrm{NJ}}^{\prime}(X)$; for the space in Example 2, $C_{\mathrm{Z}}(X)>C_{\mathrm{NJ}}^{\prime}(X)$; and for the space in Example $3, C_{\mathrm{Z}}(X)<C_{\mathrm{NJ}}^{\prime}(X)$.

We now point out another difference concerning the behaviour of $C_{\mathrm{NJ}}(X)$ and $C_{\mathrm{NJ}}^{\prime}(X)$. By replacing $x$ and $y$ by $(x+y) / 2$ and $(x-y) / 2$, respectively, one sees that for any normed space $X$ the well known equality

$$
\inf \left\{\frac{\|x+y\|^{2}+\|x-y\|^{2}}{2\left(\|x\|^{2}+\|y\|^{2}\right)}: x, y \in X, \text { not both zero }\right\}=\frac{1}{C_{\mathrm{NJ}}(X)}
$$

holds. Hence it is natural to wonder if $\inf \left\{\left(\|x+y\|^{2}+\|x-y\|^{2}\right) / 4: x, y \in S_{X}\right\}$ coincides with $1 / C_{\mathrm{NJ}}^{\prime}(X)$. This is true if $X$ is an inner product space or a non-(UNS) space. Example 2 shows that, in general, the equality is false: for $x=(-1 / 3,1)$ and $y=(-1,1 / 3)$ one gets $\left(\|x+y\|^{2}+\|x-y\|^{2}\right) / 4=2 / 3$ $<4 /(3+2 \sqrt{2})=1 / C_{\mathrm{NJ}}^{\prime}(X)$.

The characteristic of convexity of a Banach space $X$ is defined as $\varepsilon_{0}(X)=$ $\sup \left\{\varepsilon: \delta_{X}(\varepsilon)=0\right\}$. E. M. Mazcuñán-Navarro [15] proved that for any Banach space $X, C_{\mathrm{NJ}}(X) \geq 1+\varepsilon_{0}(X)^{2} / 4$. From (14) it follows immediately that also

$$
C_{\mathrm{NJ}}^{\prime}(X) \geq 1+\varepsilon_{0}(X)^{2} / 4 .
$$

For the space in Example 1, $C_{\mathrm{NJ}}^{\prime}(X)=5 / 4$, and it is easy to see that $\varepsilon_{0}(X)=1$. This shows that the above inequality is sharp.

5. Some examples. In this section we shall compute the value of $C_{\mathrm{NJ}}^{\prime}(X)$ for some spaces $X$. We shall complete the section with a table where the values of $C_{\mathrm{NJ}}(X)$ and $J(X)$, and the respective references, are also included.

For $x=\left(x_{1}, x_{2}\right) \in \mathbb{R}^{2}$, let

$$
\|x\|_{\infty}=\max \left\{\left|x_{1}\right|,\left|x_{2}\right|\right\}, \quad\|x\|_{1}=\left|x_{1}\right|+\left|x_{2}\right|, \quad\|x\|_{2}=\sqrt{x_{1}^{2}+x_{2}^{2}} .
$$

Example 1. Let $X$ be $\mathbb{R}^{2}$ endowed with the norm

$$
\|x\|= \begin{cases}\|x\|_{\infty} & \text { if } x_{1} x_{2} \leq 0 \\ \|x\|_{1} & \text { if } x_{1} x_{2} \geq 0\end{cases}
$$


Then $C_{\mathrm{NJ}}^{\prime}(X)=5 / 4$ and it is attained only at the points $\pm x$ and $\pm y$ when $x$ and $y$ are two adjacent vertices of $S_{X}$ (that is affine to a regular hexagon).

Proof. To compute $C_{\mathrm{NJ}}^{\prime}(X)$ we can consider only the following two cases according to the position of $x=\left(x_{1}, x_{2}\right)$ and $y=\left(y_{1}, y_{2}\right)$ in $S_{X}$.

CASE 1: Assume that $x=\left(x_{1}, 1\right)$ and $y=\left(y_{1}, 1\right)$ with $0 \leq x_{1} \leq y_{1} \leq 1$. Then $\|x+y\|^{2}+\|x-y\|^{2}=4+\left|x_{1}-y_{1}\right|^{2} \leq 5$, and the equality is attained only at the points $x=(0,1), y=(1,1)$.

CASe 2: Assume that $x=\left(x_{1}, 1\right)$ and $y=\left(1, y_{2}\right)$ with $0 \leq x_{1} \leq y_{2} \leq 1$. Then $\|x+y\|^{2}+\|x-y\|^{2}=\left(1+y_{2}\right)^{2}+\left(2-x_{1}-y_{2}\right)^{2}=5+2\left(x_{1}+y_{2}\right)\left(y_{2}-1\right)$ $+x_{1}\left(x_{1}-2\right) \leq 5$, and the equality is attained only at the points $x=(0,1)$, $y=(1,1)$.

EXAmple 2. Let $X$ be $\mathbb{R}^{2}$ endowed with the norm

$$
\|x\|= \begin{cases}\|x\|_{2} & \text { if } x_{1} x_{2} \geq 0 \\ \|x\|_{\infty} & \text { if } x_{1} x_{2} \leq 0\end{cases}
$$

Then $C_{\mathrm{NJ}}^{\prime}(X)=(3+2 \sqrt{2}) / 4 \approx 1.457$, and it is attained only at the points $x= \pm(1 / \sqrt{2}, 1 / \sqrt{2}), y= \pm(-1,1)$.

Proof. First, observe that for $x \in X$,

$$
\frac{1}{\sqrt{2}}\|x\| \leq\|x\|_{\infty} \leq\|x\| \leq\|x\|_{2} \leq \sqrt{2}\|x\| .
$$

To compute $C_{\mathrm{NJ}}^{\prime}(X)$ we can consider only the following four cases according to the position of $x=\left(x_{1}, x_{2}\right)$ and $y=\left(y_{1}, y_{2}\right)$ in $S_{X}$.

CASE 1: Assume that $x_{1} x_{2}, y_{1} y_{2} \geq 0$. Then $\|x\|_{2}=\|y\|_{2}=1$ and

$$
\|x+y\|^{2}+\|x-y\|^{2} \leq\|x+y\|_{2}^{2}+\|x-y\|_{2}^{2}=4<3+2 \sqrt{2} .
$$

CASE 2: Assume that $x_{1}, x_{2} \geq 0$ and $y=\left(y_{1}, 1\right)$ with $-1 \leq y_{1} \leq 0$. Then $\|x\|_{2}=\|y\|_{\infty}=1$ and $\|x-y\|=\|x-y\|_{\infty}=x_{1}-y_{1}$. If $x_{1}+y_{1} \geq 0$ then $\|x+y\|=\|x+y\|_{2}$ and therefore

$$
\begin{aligned}
\|x+y\|^{2}+\|x-y\|^{2} & =x_{1}^{2}+x_{2}^{2}+2 y_{1}^{2}+1+x_{1}^{2}+2 x_{2}=2+2 y_{1}^{2}+x_{1}^{2}+2 x_{2} \\
& \leq 2+3 x_{1}^{2}+2 x_{2}=5-3 x_{2}^{2}+2 x_{2}<3+2 \sqrt{2} .
\end{aligned}
$$

Conversely, if $x_{1}+y_{1}<0$ then $\|x+y\|=\|x+y\|_{\infty}=x_{2}+1$ and therefore

$$
\begin{aligned}
\|x+y\|^{2}+\|x-y\|^{2} & =x_{1}^{2}+x_{2}^{2}+1+2 x_{2}-2 x_{1} y_{1}+y_{1}^{2} \\
& =2+2 x_{2}-2 x_{1} y_{1}+y_{1}^{2} \leq 2+2 x_{2}+2 x_{1}+y_{1}^{2} \\
& \leq 3+2\left(x_{1}+x_{2}\right) \leq 3+2 \sqrt{2} .
\end{aligned}
$$

CASE 3: Assume that $x=\left(x_{1}, 1\right), y=\left(y_{1}, 1\right)$ with $-1 \leq x_{1}, y_{1} \leq 0$. Then $\|x+y\|=\|x+y\|_{\infty}=2$ and $\|x-y\|=\left|x_{1}-y_{1}\right|$ and therefore

$$
\|x+y\|^{2}+\|x-y\|^{2}=4+\left|x_{1}-y_{1}\right|^{2} \leq 5<3+2 \sqrt{2} .
$$


CASE 4: Assume that $x=\left(x_{1}, 1\right), y=\left(-1, y_{2}\right)$ with $-1 \leq x_{1} \leq 0$ and $0 \leq y_{2} \leq 1$. Then $\|x+y\|=\|x+y\|_{\infty}$ and $\|x-y\|=\|x-y\|_{2}$ and therefore

$$
\begin{aligned}
\|x+y\|^{2}+\|x-y\|^{2} & =\max \left\{\left(1-x_{1}\right)^{2},\left(1+y_{2}\right)^{2}\right\}+\left(1+x_{1}\right)^{2}+\left(1-y_{2}\right)^{2} \\
& =\max \left\{2\left(1+x_{1}^{2}\right)+\left(1-y_{2}\right)^{2}, 2\left(1+y_{2}^{2}\right)+\left(1+x_{1}\right)^{2}\right\} \\
& \leq 4+\max \left\{\left(1-y_{2}\right)^{2},\left(1+x_{1}\right)^{2}\right\} \leq 5<3+2 \sqrt{2} .
\end{aligned}
$$

Finally, it follows from the above that the equality $\|x+y\|^{2}+\|x-y\|^{2}$ $=3+2 \sqrt{2}$ is only possible in Case 2 and when $x=(1 / \sqrt{2}, 1 / \sqrt{2})$ and $y=(-1,1)$.

EXAmple 3. Let $X$ be $\mathbb{R}^{2}$ endowed with the norm

$$
\|x\|= \begin{cases}\|x\|_{2} & \text { if } x_{1} x_{2} \geq 0 \\ \|x\|_{1} & \text { if } x_{1} x_{2} \leq 0\end{cases}
$$

Then $C_{\mathrm{NJ}}^{\prime}(X)=3 / 2$, and it is attained only at the points $x=( \pm 1,0)$, $y=( \pm 0,1)$.

Proof. Note that for $x \in X$,

$$
\frac{1}{\sqrt{2}}\|x\| \leq\|x\|_{2} \leq\|x\| \leq\|x\|_{1} \leq \sqrt{2}\|x\| .
$$

Since $S_{X}$ is symmetric with respect to the lines $x_{2}= \pm x_{1}$, to compute $C_{\mathrm{NJ}}^{\prime}(X)$ we can consider only the following three cases according to the position of $x=\left(x_{1}, x_{2}\right)$ and $y=\left(y_{1}, y_{2}\right)$ in $S_{X}$.

CASE 1: Assume that $x_{1}, x_{2}, y_{1}, y_{2} \geq 0$. Then $\|x\|_{2}=\|y\|_{2}=1,\|x+y\|=$ $\|x+y\|_{2}$ and $\|x-y\|=\|x-y\|_{1}$. Therefore

$$
\begin{aligned}
\|x+y\|^{2}+\|x-y\|^{2} & =\|x+y\|_{2}^{2}+\left(\left|x_{1}-y_{1}\right|+\left|x_{2}-y_{2}\right|\right)^{2} \\
& =\|x+y\|_{2}^{2}+\|x-y\|_{2}^{2}+2\left|x_{1}-y_{1}\right|\left|x_{2}-y_{2}\right| \\
& =4+2\left|x_{1}-y_{1}\right|\left|x_{2}-y_{2}\right| \leq 6 .
\end{aligned}
$$

CASE 2: Assume that $1 / \sqrt{2} \leq x_{2} \leq x_{1} \leq 1$ and $1 / 2 \leq-y_{1} \leq y_{2} \leq 1$. Then $\|x\|=\|x\|_{2},\|y\|=\|y\|_{1}$. Since $x_{1}+y_{1}=x_{1}+y_{2}-1 \geq x_{1}-1 / 2>0$ and $x_{2}+y_{2}>0$, we have $\|x+y\|=\|x+y\|_{2}$. If $x_{2}-y_{2} \geq 0$, then

$$
\begin{aligned}
\|x+y\|^{2}+\|x-y\|^{2} & =\|x+y\|_{2}^{2}+\|x-y\|_{2}^{2}=2\left(\|x\|_{2}^{2}+\|y\|_{2}^{2}\right) \\
& \leq 2\left(\|x\|^{2}+\|y\|^{2}\right) \leq 4<6 .
\end{aligned}
$$

Conversely, if $x_{2}-y_{2} \leq 0$, then

$$
\begin{aligned}
\|x+y\|^{2}+\|x-y\|^{2} & =\|x+y\|_{2}^{2}+\|x-y\|_{1}^{2} \\
& =2\left(x_{1}^{2}+x_{2}^{2}\right)+2\left(y_{1}^{2}+y_{2}^{2}+\left|x_{1}-y_{1}\right|\left|x_{2}-y_{2}\right|\right) \\
& =4+2 x_{1}\left(1-x_{2}\right)+2 y_{1}\left(y_{2}+x_{1}+x_{2}\right) \\
& \leq 4+2 x_{1}\left(1-x_{2}\right) \leq 6 .
\end{aligned}
$$


CASE 3: Assume that $x_{1} \leq 0 \leq x_{2}$ and $y_{1} \leq 0 \leq y_{2}$. Then

$$
\begin{aligned}
\|x+y\|^{2}+\|x-y\|^{2} & =\|x+y\|_{1}^{2}+\|x-y\|_{2}^{2} \\
& \leq 4+\|x-y\|_{2}^{2} \\
& =4+2\left(x_{1}-y_{1}\right)^{2} \leq 6 .
\end{aligned}
$$

Checking, in the above cases, when the inequalities can be equalities it follows that the value $\|x+y\|^{2}+\|x-y\|^{2}=6$ is attained only at the points $x=( \pm 1,0)$ and $y=(0, \pm 1)$.

EXAmple 4 . Let $X$ be $\mathbb{R}^{2}$ endowed with the norm

$$
\left\|\left(x_{1}, x_{2}\right)\right\|=\max \left\{\left|x_{1}\right|+(\sqrt{2}-1)\left|x_{2}\right|,\left|x_{2}\right|+(\sqrt{2}-1)\left|x_{1}\right|\right\} .
$$

Then $C_{\mathrm{NJ}}^{\prime}(X)=4-2 \sqrt{2}$, and it is attained only at the points $\pm x$ and $\pm y$, where $x$ and $y$ are two adjacent vertices of $S_{X}$ (that is a regular octagon).

Proof. It is known that $C_{\mathrm{NJ}}(X)=4-2 \sqrt{2}$ (see [1]). Then, taking $x=$ $(1,0)$ and $y=(1 / \sqrt{2}, 1 / \sqrt{2})$ we have

$$
\begin{aligned}
4-2 \sqrt{2} & =\frac{\|x+y\|^{2}+\|x-y\|^{2}}{4} \\
& \leq C_{\mathrm{NJ}}^{\prime}(X) \leq C_{\mathrm{NJ}}(X) \\
& =4-2 \sqrt{2} .
\end{aligned}
$$

A detailed study similar to that in the preceding examples shows that $C_{\mathrm{NJ}}^{\prime}(X)$ is attained only at the points $x$ and $y$ that are adjacent vertices of $S_{X}$. On the contrary, $C_{\mathrm{NJ}}(X)$ is attained at other points (e.g., $x=(1,0), y=$ $(0, \sqrt{2}-1))$.

Example 5. Let $X$ be the space $\ell_{p}$. Then

$$
C_{\mathrm{NJ}}^{\prime}(X)=C_{\mathrm{NJ}}(X)= \begin{cases}2^{2 / p-1} & \text { if } 1 \leq p \leq 2 \\ 2^{1-2 / p} & \text { if } p \geq 2\end{cases}
$$

Proof. The value of $C_{\mathrm{NJ}}(X)$ is well known. Since $C_{\mathrm{NJ}}^{\prime}(X) \leq C_{\mathrm{NJ}}(X)$ and $X$ contains an isometric copy of $\ell_{p}^{(2)}=\left(\mathbb{R}^{2},\|\|_{p}\right)$, to prove the equality it is enough to see that there exist unit vectors $x, y \in \ell_{p}^{(2)}$ such that

$$
\frac{\|x+y\|_{p}^{2}+\|x-y\|_{p}^{2}}{4}= \begin{cases}2^{2 / p-1} & \text { if } 1 \leq p \leq 2 \\ 2^{1-2 / p} & \text { if } p \geq 2 .\end{cases}
$$

Indeed, take $x=(1,0), y=(0,1)$ if $1 \leq p \leq 2$, and $x=\left(2^{-1 / p}, 2^{-1 / p}\right)$, $y=\left(-2^{-1 / p}, 2^{-1 / p}\right)$ if $p \geq 2$. 


\begin{tabular}{|c|c|c|c|c|c|}
\hline \multicolumn{2}{|l|}{$X$} & $C_{\mathrm{NJ}}^{\prime}(X)$ & $C_{\mathrm{NJ}}(X)$ & $J(X)$ & $C_{\mathrm{Z}}(X)$ \\
\hline \multicolumn{2}{|c|}{ non-(UNS) spaces } & 2 & 2 & 2 & 2 \\
\hline \multicolumn{2}{|c|}{ Hilbert spaces } & 1 & 1 & $\sqrt{2}$ & 1 \\
\hline \multicolumn{2}{|c|}{ Example 1: $\quad \ell_{\infty}-\ell_{1}$} & $\begin{aligned} & \frac{5}{4} \\
= & 1.25\end{aligned}$ & $\begin{array}{l}\frac{3+\sqrt{5}}{4} \\
\approx 1.309[1]\end{array}$ & $\begin{aligned} & \frac{3}{2} \\
= & 1.5[12]\end{aligned}$ & $\begin{aligned} & \frac{5}{4} \\
= & 1.25[1]\end{aligned}$ \\
\hline \multicolumn{2}{|c|}{ Example 2: $\quad \ell_{2}-\ell_{\infty}$} & $\begin{array}{l}\frac{3+2 \sqrt{2}}{4} \\
\approx 1.457\end{array}$ & $\begin{aligned} & \frac{3}{2} \\
= & 1.5[1]\end{aligned}$ & $\begin{aligned} & \frac{1+\sqrt{2}}{\sqrt{2}} \\
\approx & 1.707\end{aligned}$ & $\begin{aligned} & \frac{3}{2} \\
= & 1.5[1]\end{aligned}$ \\
\hline \multicolumn{2}{|c|}{ Example 3: $\quad \ell_{2}-\ell_{1}$} & $\begin{aligned} & \frac{3}{2} \\
= & 1.5\end{aligned}$ & $\begin{aligned} & \frac{3}{2} \\
= & 1.5[1]\end{aligned}$ & $\begin{aligned} & \frac{\sqrt{8}}{\sqrt{3}} \\
\approx & 1.633[12]\end{aligned}$ & $\begin{array}{c}\sqrt{2} \\
\approx 1.414[1]\end{array}$ \\
\hline \multicolumn{2}{|c|}{ Example 4: Octagon } & $\begin{array}{l}4-2 \sqrt{2} \\
\approx 1.172\end{array}$ & $\begin{array}{l}4-2 \sqrt{2} \\
\approx 1.172\end{array}$ & $\begin{array}{c}\sqrt{2} \\
\approx 1.414[1]\end{array}$ & $\begin{array}{l}4-2 \sqrt{2} \\
\approx 1.172[1]\end{array}$ \\
\hline \multirow{2}{*}{ Example $5: \ell_{p}$} & $1 \leq p \leq 2$ & $2^{2 / p-1}$ & $2^{2 / p-1}$ & $2^{1 / p} \quad[2]$ & $2^{2 / p-1}$ \\
\hline & $p \geq 2$ & $2^{1-2 / p}$ & $2^{1-2 / p}$ & $2^{1-1 / p} \quad[2]$ & $2^{1-2 / p}$ \\
\hline
\end{tabular}

6. Passing to the dual. The examples in the previous section are relevant because they imply some interesting facts. Let $X^{*}$ denote the dual space of $X$. It is well known that $C_{\mathrm{NJ}}(X)=C_{\mathrm{NJ}}\left(X^{*}\right)$ (see, e.g., [14]). On the contrary, the value on a space is in general different from that on its dual for the constants $J(X)[12]$ and $C_{\mathrm{Z}}(X)$ [1]. This is the case for the spaces (dual to each other) in Examples 2 and 3 ; this also shows that, in general, $C_{\mathrm{NJ}}^{\prime}(X) \neq$ $C_{\mathrm{NJ}}^{\prime}\left(X^{*}\right)$. Since those spaces are reflexive (in fact, two-dimensional) they show that passing to the dual the value of the three constants can increase and decrease as well.

We can ask how big the difference can be for the different constants. Concerning $J(X)$ no sharp inequality seems to be known. We can only deduce an estimate by using the inequalities (see [12, Theorem 1])

$$
2 J(X)-2 \leq J\left(X^{*}\right) \leq \frac{J(X)}{2}+1 .
$$

From (19) we obtain the estimate $J\left(X^{*}\right)-J(X) \leq 1-J(X) / 2 \leq 1-\sqrt{2} / 2$, and a similar one for $J(X)-J\left(X^{*}\right)$. But probably this estimate is not sharp.

Now we shall give an estimate concerning the difference of the values of the constant $C_{\mathrm{NJ}}^{\prime}$ on $X$ and on $X^{*}$. 
TheOREM 4. For any normed space $X$,

$$
\begin{aligned}
C_{\mathrm{NJ}}^{\prime}(X) / 2+\sqrt{C_{\mathrm{NJ}}^{\prime}(X)-1} & \leq C_{\mathrm{NJ}}^{\prime}\left(X^{*}\right) \\
& \leq 2\left(1+C_{\mathrm{NJ}}^{\prime}(X)-\sqrt{\left.2 C_{\mathrm{NJ}}^{\prime}(X)\right)} .\right.
\end{aligned}
$$

Proof. From the inequalities $C_{\mathrm{NJ}}^{\prime}(X) \leq C_{\mathrm{NJ}}(X),(7)$ and (5), we obtain

$$
\begin{aligned}
C_{\mathrm{NJ}}^{\prime}(X) / 2+\sqrt{C_{\mathrm{NJ}}^{\prime}(X)-1} & \leq C_{\mathrm{NJ}}(X) / 2+\sqrt{C_{\mathrm{NJ}}(X)-1} \\
& =C_{\mathrm{NJ}}\left(X^{*}\right) / 2+\sqrt{C_{\mathrm{NJ}}\left(X^{*}\right)-1} \\
& \leq C_{\mathrm{NJ}}^{\prime}\left(X^{*}\right) \leq C_{\mathrm{NJ}}\left(X^{*}\right)=C_{\mathrm{NJ}}(X) \\
& \leq 2\left(1+C_{\mathrm{NJ}}^{\prime}(X)-\sqrt{\left.2 C_{\mathrm{NJ}}^{\prime}(X)\right) .} .\right.
\end{aligned}
$$

Note that both inequalities in (20) are sharp if $X$ is non-(UNS), the left one is sharp for the space in Example 3, and the right one is sharp for the space in Example 2.

COROllary 3. For any normed space $X$,

$$
\left|C_{\mathrm{NJ}}^{\prime}(X)-C_{\mathrm{NJ}}^{\prime}\left(X^{*}\right)\right| \leq 3-2 \sqrt{2} .
$$

Proof. From (20) it follows that

$$
\begin{aligned}
C_{\mathrm{NJ}}^{\prime}(X)-C_{\mathrm{NJ}}^{\prime}\left(X^{*}\right) & \leq C_{\mathrm{NJ}}^{\prime}(X)-\max \left\{1, C_{\mathrm{NJ}}^{\prime}(X) / 2+\sqrt{C_{\mathrm{NJ}}^{\prime}(X)-1}\right\} \\
& =\min \left\{C_{\mathrm{NJ}}^{\prime}(X)-1, C_{\mathrm{NJ}}^{\prime}(X) / 2-\sqrt{C_{\mathrm{NJ}}^{\prime}(X)-1}\right\} .
\end{aligned}
$$

Now, since the functions $f_{1}(t)=t-1$ and $f_{2}(t)=t / 2-\sqrt{t-1}$ are, respectively, increasing and decreasing in $[1,2]$, we see that for $\bar{t}=4-2 \sqrt{2}$, $f_{1}(\bar{t})=f_{2}(\bar{t})=3-2 \sqrt{2} \geq \min _{1 \leq t \leq 2}\left\{f_{1}(t), f_{2}(t)\right\}$, and we conclude that $C_{\mathrm{NJ}}^{\prime}(X)-C_{\mathrm{NJ}}^{\prime}\left(X^{*}\right) \leq 3-2 \sqrt{2}$. On the other hand, (20) yields

$$
C_{\mathrm{NJ}}^{\prime}\left(X^{*}\right)-C_{\mathrm{NJ}}^{\prime}(X) \leq 2+C_{\mathrm{NJ}}^{\prime}(X)-2 \sqrt{2 C_{\mathrm{NJ}}^{\prime}(X)} \leq 3-2 \sqrt{2}
$$

\section{References}

[1] J. Alonso and P. Martín, A counterexample to a conjecture of G. Zbăganu about the Neumann-Jordan constant, Rev. Roumaine Math. Pures Appl. 51 (2006), 135-141.

[2] E. Casini, About some parameters of normed linear spaces, Atti Accad. Lincei Rend. Cl. Sci. Fis. Mat. Nat. 80 (1986), 11-15.

[3] J. A. Clarkson, The von Neumann-Jordan constant for the Lebesgue spaces, Ann. of Math. 38 (1937), 114-115.

[4] M. M. Day, Uniform convexity in factor and conjugate spaces, ibid. (2) 45 (1944), 375-385.

[5] —, Some characterizations of inner product spaces, Trans. Amer. Math. Soc. 62 (1947), 320-337.

[6] T. Figiel, On moduli of convexity and smoothness, Studia Math. 56 (1976), 121-155.

[7] J. Gao, Normal structure and Pythagorean approach in Banach spaces, Period. Math. Hungar. 51 (2005), 19-30.

[8] -, A note on Pythagorean approach in Banach spaces, J. Dynam. Systems Geom. Theor. 4 (2006), 125-132. 
[9] J. Gao, A Pythagorean approach in Banach spaces, J. Inequal. Appl. 2006, Art. ID 94982, 11 pp.

[10] -, On some geometric parameters in Banach spaces, J. Math. Anal. Appl. 334 (2007), 114-122.

[11] H. Ishihara and W. Takahashi, Modulus of convexity, characteristic of convexity and fixed point theorems, Kodai Math. J. 10 (1987), 197-208.

[12] M. Kato, L. Maligranda and Y. Takahashi, On James and Jordan-von Neumann constants and the normal structure coefficient of Banach spaces, Studia Math. 144 (2001), 275-295.

[13] M. Kato and K.-I. Miyazaki, Remark on generalized Clarkson's inequalities for extreme cases, Bull. Kyushu Inst. Tech. Math. Natur. Sci. 41 (1994), 27-31.

[14] M. Kato and Y. Takahashi, On the von Neumann-Jordan constant for Banach spaces, Proc. Amer. Math. Soc. 25 (4) (1997), 1055-1062.

[15] E. M. Mazcuñan-Navarro, Banach space properties sufficient for normal structure, J. Math. Anal. Appl. 337 (2008), 197-218.

[16] L. Y. Nikolova, L. E. Persson and T. Zachariades, A study of some constants for Banach spaces, C. R. Acad. Bulg. Sci. 57 (2004), 5-9.

[17] P. L. Papini, Constants and symmetries in Banach spaces, Ann. Univ. Mariae CurieSkłodowska Sect. A 56 (2002), 65-76.

[18] A. I. Perov, Potential operators, Math. Notes 24 (1978), 793-799.

[19] S. Saejung, On James and von Neumann-Jordan constants and sufficient conditions for the fixed point property, J. Math. Anal. Appl. 323 (2006), 1018-1024.

[20] —, Sufficient conditions for uniform normal structure of Banach spaces and their duals, ibid. 330 (2007), 597-604.

[21] Y. Takahashi, Some geometric constants of Banach spaces-A unified approach, Abstract, Conference Katakyushu 9/06.

[22] C. Yang and F. Wang, On a new geometric constant related to the von NeumannJordan constant, J. Math. Anal. Appl. 324 (2006), 555-565.

[23] G. Zbăganu, An inequality of $M$. Rădulescu and S. Rădulescu which characterizes inner product spaces, Rev. Roumaine Math. Pures Appl. 47 (2001), 253-257.

Departamento de Matemáticas

Universidad de Extremadura

Avda. de Elvas s/n

06071 Badajoz, Spain

E-mail: jalonso@unex.es pjimenez@unex.es
Dipartimento di Matematica Università di Bologna Piazza Porta S. Donato, 5 40126 Bologna, Italy E-mail: pierluigi.papini@unibo.it

Received September 29, 2007

Revised version May 12, 2008 